\title{
Recombinant DNA technology as a tool for improving production of polyhydroxyalkanoates by the natural producers
}

\author{
Daniela Chmelová ${ }^{\bowtie}$, Barbora Legerská and Miroslav Ondrejovič \\ Department of Biotechnology, Faculty of Natural Sciences, University of SS. Cyril and Methodius in Trnava, Nám. J. Herdu 2, \\ Trnava, SK- 917 01, Slovak Republic
}

\author{
Article info \\ Article history: \\ Received: $12^{\text {th }}$ October 2020 \\ Accepted: $24^{\text {th }}$ November 2020

Keywords:
Aeromonas
Bacillus
Cupriavidus
Halomonas
Polyhydroxyalkanoates
Pseudomonas
Recombinant
Abbreviations:
CRISPR - clustered regularly interspaced
$\quad$ short palindromic repeats
HPH - high pressure homogenization
MscL - mechanosensitive channels of large
PHA - ponductance
TCA - tricarboxylic acid cycle \\ Keywords: \\ Bacillus \\ Cupriavidus \\ xyalkanoates \\ Pseudomonas \\ Abbreviations: \\ CRISPR - clustered regularly interspaced \\ short palindromic repeats \\ $\mathrm{HPH}$ - high pressure homogenization \\ TCA - tricarboxylic acid cycle
}

\begin{abstract}
Polyhydroxyalkanoates (PHAs) are a group of the biodegradable polyesters, and represent an alternative to conventionally used petroleum-based plastics resistant to biodegradation. The production is not cost-competitive compared to conventional plastics, although, there are several bacterial producers capable of PHA accumulating up to $80 \%$ of their cells dry weight using low-cost substrates. PHA production can be improved by transferring specific enzymes or entire metabolic pathways from the most efficient producers to other natural producers. Therefore, the review is focused on genetic modification of bacterial producers, namely the genera Cupriavidus, Pseudomonas, Halomonas, Aeromonas and Bacillus, for efficient industrial production of PHAs. Recombinant PHA producer can use non-traditional substrates like agro-industrial wastes, namely whey, lignocellulose or glycerol. It is possible to influence the shape and size of the producer's cell by over-expression or knockout of selected genes or to affect its preference for a specific component of a culture medium by modulation of a producer's basal metabolism. The costs of PHA production still be reduced by simplifying the downstream process by enzymatic hydrolysis of selected parts of the cell or blocking the protective mechanisms of the cell against its autolysis caused by the ionic strength of the solution.
\end{abstract}

(C) University of SS. Cyril and Methodius in Trnava

\section{Introduction}

Polyhydroxyalkanoates (PHAs) as the microbial storage biopolymers are usually accumulated in response to the absence of a source of macroelements as oxygen, nitrogen, phosphorus, sulfur, iron or magnesium with an excess of carbon. Cupriavidus, Pseudomonas, Aeromonas, Bacillus, Alcaligenes, Enterobacter and certain cyanobacteria and halophilic bacteria produce PHAs under these conditions (Leong et al. 2014). Although some producers, namely Alcaligenes eutrophus, Azotobacter vinelandii UWD, Alcaligenes latus or recombinant Escherichia coli, are able to constantly accumulate PHAs without nutrient-limited conditions), but usually accumulate less PHA (Keshavarz and Roy 2010).

The producers usually generate short-chain length (scl) PHAs composed of monomers containing 3 to 5 carbon atoms (C. necator, A. latus), mainly polyhydroxybutyrate (PHB) or polyhydroxyvalerate (PHV), or medium-chain length (mcl) PHAs composed of monomers consisting of 6 to 14 carbon atoms (Aeromonas caviae, Pseudomonas putida) such as polyhydroxyhexanoate (PHH), polyhydroxyoctanoate (PHO), polyhydroxydecanoate (PHD) or polyhydroxydodecanoate (PHDD). PHA producers rarely accumulate long- 
chain length (lcl) PHAs composed of monomers containing more than 15 carbon atoms (Zhang et al. 2006; Tan et al. 2014b).

The material properties of PHAs are similar to those of conventional synthetic petroleum-based plastics and therefore, represent a suitable alternative made from renewable resources (Tian et al. 2005; Berezina and Martelli 2014; Troschl et al. 2017). Moreover, these biopolymers are fully biodegradable into carbon dioxide and water by microorganisms from a variety of artificial and natural environment, including wastewater, soil, rivers, lakes, seas, and even hot springs and polar regions (Chen et al. 2018; Favaro et al. 2019; Tan et al. 2020).

Despite the above-mentioned advantages, the cost of PHA production remains high compared to traditional plastics (Koller et al. 2013). The aim of the research is therefore to find a fast and cheap way to produce PHAs. Researchers have previously focused on finding a suitable and low-priced substrate for the production of specific PHAs, including the use of by-products derived from a production process as a source of carbon and energy. Current research is concerned with the metabolic improvement of selected species, either in terms of influencing metabolic pathways leading to PHA accumulation, expanding the range of substrates used by the producers or facilitating isolation of PHAs from the cell. In this review, we summarize the latest knowledge about the possibilities of increasing PHA production by genetically modified PHA producers of the genera Cupriavidus, Pseudomonas, Halomonas, Aeromonas and Bacillus.

\section{Genetically modified PHA producers}

\section{Genetic modifications for inexpensive carbon sources utilization}

Selection of carbon source is one of the important factors that influence the price of a PHA product. It must be cheap and available in large quantities (Choi and Lee 1997). By-products from various industrial or agricultural productions are strong candidates for this purpose (Koller et al. 2005; Solaiman et al. 2006). However, a number of PHAaccumulating bacterial producers cannot use them as a substrate, but this can be changed through genetic engineering.

Whey high in lactose, proteins and lipids as a byproduct of milk processing is available in large quantities, but only some strains from the group of PHA producers can utilize it, namely Hydrogenophaga pseudoflava DSM1034 (Koller et al. 2007), Methylobacterium sp. ZP24 (Yellore and Desai 1998) and Thermus thermophilus HB8 (Pantazaki et al. 2009). Therefore, the induction of the lac $Z$ gene coding for $\beta$-galactosidase was one of the first attempts to expand the range of substrates for PHA production. Povolo et al. (2010) disrupted the phaZl (PHA depolymerase) gene of the $C$. necator wild-type strain by the lacZ gene replacement with the aim of producing PHAs in a medium with lactose as the sole carbon source. In contrast to the wild-type $C$. necator, the recombinant strain was able to grow and produce $\mathrm{PHB}$ in a medium with lactose and whey. PHB accumulation represented $30 \%$ of cell biomass after $48 \mathrm{~h}$ in a hydrolyzed whey permeate composed mainly of glucose and galactose, and $22 \%$ of cell biomass after $48 \mathrm{~h}$ in a nonhydrolyzed permeate high in lactose. The yield of PHB probably reduced due to non-optimal cultivation conditions. However, due to the low yields of PHAs, research is focused on finding the native PHA-accumulating producers containing lactose-degrading enzyme (Favaro et al. 2019).

Molasses is another agricultural by-product of sugarcane or sugar beet processing in the sugar industry. The material contains sucrose usable as the sole carbon source for a PHA producer with the sucrose-degrading enzyme system. If this is not the case, then a gene encoding $\beta$-fructofuranosidase, an enzyme that cleaves sucrose to glucose and fructose, can be inserted into the genome of a PHA producer. Park et al. (2015) inserted this gene into the $C$. necator wild-type strain to produce high content of PHB (73.2 wt \%) from molasses. The conversion of byproducts from oil processing has also been achieved with genetically modified strains. An example is the utilization of glycerol as a carbon source for PHA production. Cardozo et al. (2019) inserted the $b m g D$ gene encoding glycerol3-phosphate dehydrogenase into the Bacillus megaterium PHA producer, and observed 
a higher biomass yield and PHA content compared to the wild-type producer.

Lignocellulose is one of the promising widely available and renewable sources of carbon and energy. Hydrolysates of various plants have previously been used as the substrates for PHA production. In hydrolysates, the target substrate for PHA production was glucose as a hydrolytic product released from cellulose. Then, research focused on the utilization of hemicellulose degradation products by incorporating genes required for the consumption of arabinose (Lu et al. 2013) or xylose (Liu et al. 2014) into C. necator. Although these carbohydrates can be used as substrates, lignocellulosic material is generally difficult to use in fermentation processes due to the complicated structure and compounds with a potentially toxic effect on bacterial growth. Phenolic compounds linked to lignocellulose via ester bonds pose a challenge for bioproduct conversion. They are released from lignin as well as hemicelluloses and significantly inhibited the growth of a wide range of microorganisms (Graf and Altenbuchner 2014; Bouarab-Chibane et al. 2019). Currently, attempts to assimilate phenols from lignocellulose to valuable products are known. Zhou et al. (2020) focused on the use of ferulic acid for PHA production. Although the PHA producer of $P$. putida KT2440 has natural ability to convert ferulic acid to mcl-PHA, the organism's low tolerance to ferulic acid and its toxicity complicate fermentation process. Zhou et al. (2020) modified the genome of $P$. putida KT2440 using a CRISPR/Cas9 (clustered regularly interspaced short palindromic repeats) genomeediting strategy and via this method, nine genes involved in ferulic acid-to PHA bioconversion were integrated into the $P$. putida genome. The recombinant strain increased the production of PHA to $270 \mathrm{mg} . \mathrm{L}^{-1}$ associated with the utilization of $20 \mathrm{mg} . \mathrm{L}^{-1}$ ferulic acid from a medium.

\section{Genetic modifications for production of PHA in co-cultivation system}

Co-cultivation represents an interesting strategy for the simultaneous cultivation of two or more organisms. The metabolic product of the first producer becomes a substrate for another in order to produce the desired metabolite and also utilize a by-product (Goldford et al. 2018; Fedeson et al. 2020). This concept occurs naturally in the environment, meaning various types of bacteria in communities form a network of interspecies interactions. Therefore, the strategy appears to be a suitable alternative for the utilization of difficultto-use carbon sources for PHA producers. The co-cultivation system for PHA production usually consists of two bacterial strains (Fedeson et al. 2020; Hobmeier et al. 2020; Liu et al. 2020).

Hobmeier et al. (2020) tested the possibility of mcl-PHA production in a co-culture of Synechococcus elongatus cscB with P. putida. S. elongatus fixes $\mathrm{CO}_{2}$ and metabolizes it to sucrose at high salt concentrations and then $P$. putida metabolizes sucrose and accumulates mcl-PHA in the cytoplasm. However, S. elongatus requires a nitrate-containing medium for efficient sucrose production and $P$. putida can convert nitrate to ammonium via nitrite that uses as a nitrogen source for its growth. This is a challenge because the PHA production by $P$. putida is more profitable under nitrogen-limited conditions. The recombinant strain of $P$. putida with a deletion of the nasT gene lost the ability to convert nitrate as a nitrogen source, but the ability to grow in a medium with ammonium was not affected. The mutant was able to produce up to 8.8 -fold higher PHA yield $(14.8 \%)$ in the co-culture compared to the wildtype strain.

Co-cultivation system consisting of recombinant bacterial species of $S$. elongatus and $P$. putida was also used in the work of Fedeson et al. (2020). The aim was the simultaneous production of mclPHAs and the bioremediation of toxic 2,4-dinitrotoluene (2,4-DNT). The recombinant sucrose-uptake $P$. putida (Hobmeier et al. 2020) was further modified by insertion of the $d n t A$ and $d n t B$ genes from Burkholderia sp. R34 allowing the degradation of 2,4-DNT to form nontoxic metabolites of nitrite, pyruvate and propionylCoA. This co-cultivation resulted in the degradation of 2,4-DNT associated with the production of mcl-PHA. Although, a lower mcl-PHA yield compared to the results of the other studies (Yang et al. 2019; Hobmeier et al. 2020) was observed, the advantage is the combination of biopolymer production and bioremediation thus extending the possibility of using co-cultivation strategies. 
Liu et al. (2020) verified the possibility of coculture system consisting of recombinant $E$. coli and $P$. putida strains. The production of mcl-PHA was stimulated by the presence of acetate produced by recombinant $E$. coli. Moreover, E. coli was modified by knockout of the pts $G, \operatorname{manZ}$, atpFH and $e n v R$ genes resulting in the preferred carbon source being xylose (not glucose) and $P$. putida had an amplified acetate assimilation pathway by overexpression of acetyl-CoA synthetase and acetate kinase-phosphotransacetylase (Yang et al. 2019). The growth of $P$. putida was ensured by glucose as a carbon source and mcl-PHA production by acetate produced by the recombinant $E$. coli strain. The highest mcl-PHA yield was observed by using a 20 g. $\mathrm{L}^{-1}$ carbon source (glucose : xylose, 1 : 1 ; w/w), namely 0.541 g. $\mathrm{L}^{-1}$, which is 3.9 -fold higher compared to $P$. putida monoculture.

\section{Morphology engineering for improving PHA production}

Morphology engineering is an interesting approach to increase the efficiency of PHA production without interfering the metabolic pathways of PHA production. This type of engineering affects the morphology of the cell and allows the formation of larger PHA granules, which should also facilitate their isolation. A number of genes are responsible for the shape of the cells, and by modifying them, it is possible to change the shape of the cell from rods to fibers or to form large from small spheres. For example, the $\min C D$ gene is responsible for cell length, the $f t s Z$ gene for the production of cell division protein and the $m r e B$ gene for cytoskeleton protein production (Jiang and Chen 2016; Elhadi et al. 2016; Zhao et al. 2019).

Over-expression of the $m r e B$ gene led to the formation of larger cells compared to the control (Wu et al. 2016), but over-expression of this gene associated with knockout of the $\min C D$ gene increased mcl-PHA production in $P$. mendocina NKU (Zhao et al. 2019). However, this strategy was more successful in the process of producing PHB by the recombinant $E$. coli strain (Elhadi et al. 2016). Tan et al. (2014a) observed that the inducible over-expression of the $\min C D$ gene during the stationary growth phase of Halomonas
TD08 elongated cell shape, leading to 1.2-fold higher accumulation of PHB in the cells. Another advantage of modifying the cell shape was the formation of intertwined fiber network during the production of PHB. This network allowed the self-flocculation and precipitation of cells, simplifying the subsequent downstream processing. Jiang et al. (2017) developed a temperaturesensitive plasmid expression system containing the $m r e B$ or fts $Z$ genes and introduced this construct into $H$. campaniensis cells. Recombinants are able to grow at $30{ }^{\circ} \mathrm{C}$ and their growth is comparable to the wild-type strain. Morphological changes such as cell size expansion or cell shape elongation did not appear until the temperature was raised to $37{ }^{\circ} \mathrm{C}$ and the temperature-sensitive plasmid was no longer replicated in the recombinants. This led to an increase in PHB yield, which reached up to $80 \%$ cell dry weight. The results of the above-mentioned research show a new interesting strategy for the industrial PHA production by combining the expression and/or knockout of various metabolic and morphological genes.

It is possible to influence not only the cell morphology but also the morphology of the PHA granules themselves. The phaPl gene is responsible for regulating the size and number of PHA granules. Knockout of this gene resulted in the production of PHA granules up to $10 \mu \mathrm{m}$ accumulated in the intracellular space of recombinant Halomonas bluephagenesis TD01 cells. The resulting size was limited only by the size of the cell itself (Shen et al. 2019).

\section{Genetic modifications for improving PHA biosynthesis}

PHB synthesis is one of the most studied metabolic pathways of PHAs. It consists of three basic enzymatic steps including enzymes such as PhaA ( $\beta$-ketothiolase), PhaB (NADPH-dependent acetoacetyl-CoA reductase) and PhaC (PHA synthase) (Fig. 1). PhaA catalyzes the condensation of two acetyl-CoA molecules to acetoacetyl-CoA that is converted to $(R)-3-\mathrm{HB}-\mathrm{CoA} \quad((R)-3-$ hydroxybutyryl-CoA) by $\mathrm{PhaB}$. In the final step, PhaC polymerizes $(R)-3$-HB-CoA into PHB. PhaC belongs to the key enzymes for PHA biosynthesis and catalyzes the polymerization of hydroxyacyl- 
CoA thioesters. The other PHA biosynthetic pathways differ mainly in the enzymes forming various hydroxyacyl-CoA thioesters for final polymerization by $\mathrm{PhaC}$.

$\beta$-oxidation of fatty acids is an alternative PHA biosynthetic pathway for the formation of hydroxyacyl thioesters (Fig. 1). The products of this pathway are mainly mcl-PHAs due to the length of formed precursors. The key enzymes are FadE (acyl-CoA dehydrogenase) and PhaJ (enoyl-CoA hydratase). FadE catalyzes the reaction of acylCoA to enoyl-CoA and PhaJ uses enoyl-CoA as a substrate. Some PHAs can be synthesized from (R)-3-hydroxyacyl-CoA obtained from 3-ketoacylCoA using FabG (3-ketoacyl-CoA reductase) (Philip et al. 2007).

The third PHA biosynthetic pathway involves two important enzymes, namely PhaG (3-hydroxyacylACP-CoA transferase) and FabD (malonyl-ACP transacylase) (Fig. 1). The substrates are converted to $(R)$-3-hydroxyacyl-ACP and then to $(R)$-3hydroxyacyl-CoA by $\mathrm{PhaG}$ and thus to PHA by PhaC (Philip et al. 2007; Laycock et al. 2013). The involvement of above-mentioned enzymes in PHA biosynthesis associated with important metabolic pathways such as Calvin, ribulose monophosphate (RuMP) or Krebs cycle, glycolysis as well as pathways for biosynthesis and degradation of amino acids and fatty acids ( $\mathrm{Lu}$ et al. 2009).

Bacteria can accumulate PHAs composed of different monomers depending on the carbon source used (Noda et al. 2005), with PhaC playing a key role in this process. The PhaC enzymes with different specificity can use variable precursors for PHA synthesis. PhaC can be classified into one of four classes (I - IV) based on primary structure, substrate specificity, and subunit composition (Rehm 2003; Pötter and Steinbüchel 2005). Recently, Tan et al. (2020) discovered a new PhaC isolated from the Antarctic Janthinobacterium isolate, which could be included in the new fifth class.

Over-expression of the phaC gene generally leads to higher yields of PHAs. Akdoğan and Çelik (2020) over-expressed the native phaC gene in $B$. megaterium, resulting in increased biomass yield and PHA content compared to the wild-type strain in both batch and fed-batch cultivation processes. The use of the phaC gene isolated from other PHA producers may lead to the incorporation of the new hydroxyacyl-CoA thioester monomers to the growing PHA chain, and moreover, knockout of the original phaC gene and incorporation of the new phaC gene can cause the formation of a completely new PHA. After insertion of the phaC gene from Pseudomonas sp. 61-3 into $C$. necator, the mutant was able to accumulate copolymer $\mathrm{P}(3 \mathrm{HB}-\mathrm{co}-3 \mathrm{HA})$ with a relatively high 3HB content (Matsusaki et al. 2000a; Matsusaki et al. 2000b; Matsumoto et al. 2001). Cha et al. (2020) tested the effect of four different phaC genes from Thiocapsa pfennigii, C. necator H16, Chromobacterium violaceum, and Paracoccus denitrificans on PHA production by $P$. putida EM42. Knockout of the phaC1 and phaC2 native genes in all four mutants resulted in the production of $\mathrm{P}(3 \mathrm{HV}-\mathrm{co}-4 \mathrm{HV})$ copolymer instead of the naturally produced mcl-PHA consisting of $3 \mathrm{HD}$ and $3 \mathrm{HO}$. The most common ratio of the accumulated scl-PHA was $60: 40(\mathrm{w} / \mathrm{w})$ for $3 \mathrm{HV}$ and $4 \mathrm{HV}$, respectively and $3 \mathrm{HB}$ content was only about $1 \%$. The mutant with the PhaC gene from $P$. denitrificans produced a copolymer with a ratio of $3 \mathrm{HV}: 4 \mathrm{HV} 96: 4$ (w/w). This synthase showed lower substrate specificity for $4 \mathrm{HV}$ compared to other synthases.

Insertion of the phaC gene from another producer may be accompanied by a negative effect on the type of produced PHA. The Aeromonas hydrophila mutant with the phaC gene from Pseudomonas stutzeri 1317 accumulated PHA consisting of C4-C12 monomers with a dominant amount of $3 \mathrm{HHx}$ instead of $\mathrm{P}(3 \mathrm{HV}-\mathrm{co}-4 \mathrm{HH})$ (Liu et al. 2011). Tan et al. (2020) used the $C$. necator mutant with the phaC genes from Antarctic Janthinobacterium isolates. No accumulation of PHA was observed in the first case and in the second case, the mutant accumulated only 18.7 wt $\%$ of $\mathrm{P}(3 \mathrm{HB})$. The higher $\mathrm{PHB}$ content was determined in biomass growing at $20{ }^{\circ} \mathrm{C}$ compared to $30{ }^{\circ} \mathrm{C}$. The key factor influencing the choice of synthase source is both the specificity of the enzyme and the differences of cultivation conditions between the PHA producer and the phaC source.

The other important enzymes for PHB biosynthesis are $\mathrm{PhaA}$ and $\mathrm{PhaB}$ responsible for the preparation of precursors for the synthesis of PHB chain (Philip et al. 2007). If $\mathrm{PhaA}$ and $\mathrm{PhaB}$ expression is too 


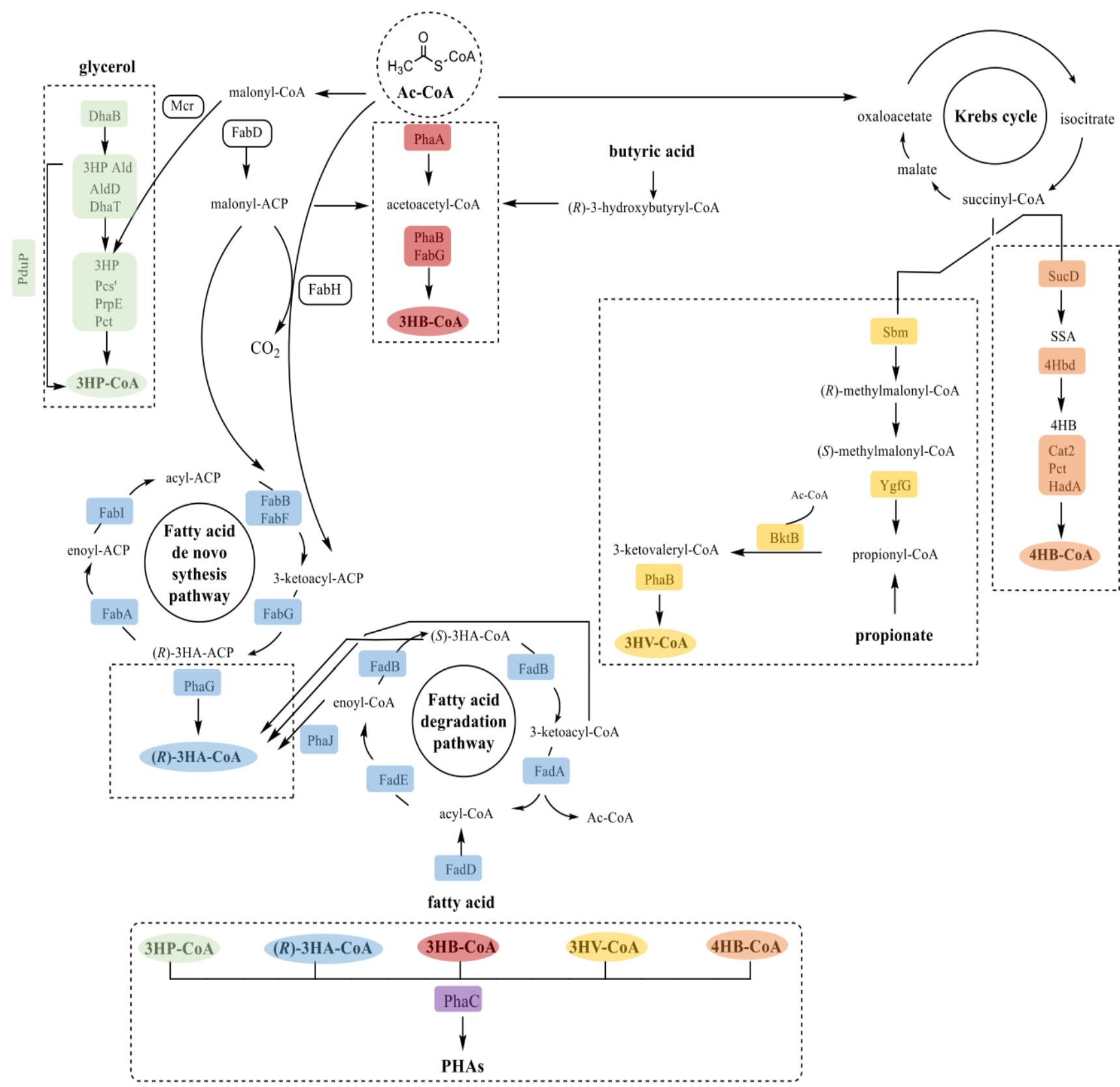

Fig. 1. Metabolic pathways of PHA production (modified from Choi et al. 2020). Legend: 3HA-CoA - 3-hydroxyacyl-CoA; 3HB - 3-hydroxybutyrate; 3HP - 3-hydroxypropionate; 3HV - 3-hydroxyvalerate; 4HbD - 4-hydroxybutyrate dehydrogenase; AldD - aldehyde dehydratase; $\mathrm{BktB}$ - $\beta$-ketothiolase; Cat2 - 4-hydroxybutyryl-CoA transferase; CoA - coenzyme A; DhaB glycerol dehydratase; DhaT - 1,3-propanediol dehydrogenase; FabA - 3-hydroxyl-ACP dehydrase; FabB - $\beta$-ketoacyl-ACP synthase I; FabD - malonyl-CoA:ACP transacylase; FabF - $\beta$-ketoacyl-ACP synthase II; FabG - 3-ketoacyl-ACP reductase; FabH - $\beta$-ketoacyl-ACP synthase III; FabI - enoyl-ACP reductase; FadA - 3-ketoacyl-CoA thiolase; FadB - 3-hydroxyacylCoA dehydrogenase; FadD - acetyl-CoA synthetase; FadE - acyl-CoA dehydrogenase; HadA - isocaprenoyl-CoA:2HIC CoAtransferase; Pcs' - propionyl-CoA synthetase; Pct - propionyl-CoA transferase; PduP - propionaldehyde dehydrogenase; PhaA - $\beta$-ketothiolase; PhaB - acetoacetylCoA reductase; PhaC - polyhydroxyalkanoate synthase; PhaD - PHApolyhydroxyalkanoate depolymerase; PhaG - 3-hydroxyacyl-ACP-CoA transferase; PhaJ - enoyl-CoA hydratase; PrpE propionyl-Coa synthetase; Sbm - methylmalonyl-CoA mutase; SucD - succinate semialdehyde dehydrogenase; YgfG methylmalonyl-CoA decarboxylase.

low and there are no other sources of metabolic in biopolymer accumulation (Liu et al. 2011). precursors for PHA synthesis, over-expression The activity of PhaA and PhaB should be checked of the phaC gene does not lead to an increase before modifying the producer's phaC gene. Over- 
expression of the phaA and phaB genes in the $A$. hydrophila mutant caused $85 \%$ increase in copolymer accumulation compared to the wildtype strain. Knockout of the acetic acid pathway was also beneficial for this strain resulting in further $47 \%$ higher accumulation of PHA copolymer due to the prevention of acetic acid formation (Liu et al. 2011).

Unlike Liu et al. (2011), Shi et al. (2020) did not reduce acetic acid production by deleting certain genes in the acetic acid pathway, but used this acid as a carbon source to produce HBT in the $A$. hydrophila mutant with over-expression of the phaA and phaB genes. Moreover, overexpression of enzymes involved in the acetic acid pathway caused further 6-fold higher PHB content compared to that produced by control strain without this modification. The high activity of PhaA and PhaB caused the accumulation of a sufficient intracellular concentration of 3-hydroxybutyryl-CoA, a key intermediate for PHB synthesis by PhaC. Subsequent overexpression of phaC led to further increase in PHA accumulation to $19.82 \%$ of cell biomass. This is the first study to report the production of PHB by engineered $A$. hydrophila in the acetatepropionate medium.

PhaB uses NADH or NADPH to catalyze its reaction. The most of $\mathrm{PHB}$-accumulating bacteria use NADPH dependent PhaB (Ling et al. 2018). NADPH is a typical cofactor for the redox reaction of anabolism. Selected species of bacteria such as Allochromatium vinosum, Azotobacter beijierinckii and $H$. bluephagenesis have NADH dependent PhaB. NADH plays a key role in respiration or anaerobic fermentation processes at high or low oxygen concentrations, respectively (Sauer et al. 2004). NADPH-dependent PhaBs are dependent on the availability of a reduced form of NADPH, which is consumed in any anabolic process in the cell and therefore, a significant accumulation of PHA occurs under essential element-limited conditions $(\mathrm{N}, \mathrm{P}, \mathrm{S}, \mathrm{Fe}$ or $\mathrm{Mg}$ ) with an excess of carbon (Steinbüchel 1991). At the same time, $\mathrm{NADH}$ is used exclusively within respiration or fermentation. Ling et al. (2018) demonstrated that $H$. bluephagenesis produced higher concentrations of NADH under oxygen-limited conditions. Blocking the etf operon limited electron transfer from $\mathrm{NADH}$, a lack of oxygen increased the $\mathrm{NADH} / \mathrm{NAD}^{+}$ratio, resulting in an increase PHB content to $90 \%$ of dry weight. PHB production under oxygen-limiting conditions represents a significant reduction in energy consumption due to the possibility of carrying out fermentation with a lower agitation rate and less aeration (Ling et al. 2018). The problem of NADH excess inhibiting pyruvate metabolism and the tricarboxylic acid (TCA) cycle was solved by adding acetic acid to the culture medium. NADH-dependent PhaB consumes NADH during the reduction of acetic acid to $3 \mathrm{HB}-\mathrm{CoA}$. Moreover, the addition of the acetic acid increased both the biomass yield and the PHB content.

Production of new copolymers can be achieved by replacing the phaB gene with genes involved in other metabolic pathways of PHA production. Jung et al. (2019) prepared the recombinant C. necator strain by deletion of the phaB1, phaB2 and phaB3 genes and over-expressing a synthetic operon consisting of phaC2 from Rhodococcus aetherivorans, phaA and phaJ from Pseudomonas aeruginosa. The recombinant strain produced the terpolymer $\mathrm{P}(3 \mathrm{HB}-\mathrm{co}-3 \mathrm{HV}-\mathrm{co}-3 \mathrm{HHx})$ (42\% of dry weight) in a medium containing volatile fatty acids such as propionate and butyrate instead of $\mathrm{P}(3 \mathrm{HB})$. Yu et al. (2020) deleted the native phaC gene in $H$. bluephagenesis and expressed the phaC and phaJ genes from A. hydrophila and endogenous fadD (acyl-CoA synthetase). The mutant produced copolymer $\mathrm{P}(3 \mathrm{HB}-\mathrm{co}-3 \mathrm{HHx})$ with the high content of $3 \mathrm{HH}(35 \%)$.

FadE and PhaJ play a key role in $\beta$-oxidation of fatty acids. Although FadE represents a ratelimiting step in $\beta$-oxidation of fatty acids, it has received little attention (Eggers and Steinbüchel 2013; Magdouli et al. 2015). PhaJ is one of the more studied enzymes because its insertion or modification makes it possible to obtain scl-mcl copolymers using a recombinant $C$. necator strain with more advantageous physical properties, such as flexibility and elasticity, compared to commonly produced PHB (Noda et al. 2005). Flores-Sánchez et al. (2020) constructed a recombinant $C$. necator with an inserted phaJl gene from $P$. putida KT2440 and a fadE gene from E. coli K12. Activation of the $f a d E$ gene caused the higher biomass yield and PHA accumulation than the original strain. The phaJl gene from $P$. putida was selected because of its high affinity for $3 \mathrm{HHx}$ 
and $3 \mathrm{HO}$ substrates. Its activation led to the implementation of $3 \mathrm{HO}$ and 3HD into the formed scl-mcl copolymer. This result supports the hypothesis that PhaC from $C$. necator can incorporate also mcl monomers into the PHA chain if suitable metabolic precursors are available.

Deletion of $p h a G$ and phaZ genes involved in $\beta$-oxidation of fatty acids causes the production of mcl-PHA with an increased content of 3HD or 3HDD monomers. Zhao et al. (2020) prepared the $P$. mendocina NK-01 mutant with deleted $p h a G$ and phaZ genes. Deletion increased the amount of 3HD (94.7 mol \%) and 3HDD (68.7 mol \%) in a medium containing sodium decanoate and sodium dodecanoate.

FadD allows efficient conversion of lipids to PHA through fatty acid metabolism (Fig. 1). Liu et al. (2011) observed that co-expression of the $v g b$ (Vitreoscilla hemoglobin gene) and fadD (Escherichia coli) genes in A. hydrophila 4AK4 led to the production of homopolyester $\mathrm{P}(3 \mathrm{HV})$ $(47.74 \%)$.

The Vitreoscilla hemoglobin ( $\mathrm{VHb}$ ) is an important oxygen-binding protein. $\mathrm{VHb}$ is induced at low concentration of oxygen in wild-type producers, effectively binding the oxygen and supplies it to the terminal respiratory oxidases, leading to respiration under oxygen-deficient conditions (Webster 1988). This mechanism affects the production of PHA by bacteria such as E. coli, A. hydrophila and P. putida (Liu et al. 2011; Duarte de Oliveira et al. 2020). Tang et al. (2020) expressed the $v g b$ gene in $C$. necator together with the knockout of the $l d h$ gene ( $L$-lactate dehydrogenase). The mutant showed higher productivity and PHB content and lower production of fermentation by-products compared to the wildtype strain in low oxygen culture medium. Ouyang et al. (2018) expressed low-oxygen inducible $\mathrm{VHb}$ in PHA producers of $H$. bluephagenesis TD01 and $H$. campaniensis LS21. Both mutants achieved more intensive biomass growth and higher PHB production. However, Duarte de Oliveira et al. (2020) observed that the production of VHb did not mitigate the negative effect of oxygen limitation. The efficiency of $\mathrm{VHb}$ engineering must be assessed for each producer. There is a possible explanation for these different results. While Duarte de Oliveira et al. (2020) focused on the producers of mcl-PHA derived from precursors of $\beta$-oxidation of fatty acids, Tang et al. (2020) worked with the producers of scl-PHA derived from precursors of glycolysis. Duarte de Oliveira et al. (2020) also observed the lower biomass yield and PHA content compared to the wild-type strain. These results suggest that an incorrectly selected metabolic modification may cause an increase in the metabolic load of the cell, resulting in a decrease in PHA production.

3-hydroxyacyls are commonly produced, but it is also possible to enrich the biopolymer with 4-hydroxyacyls by inserting the orfZ gene (4HBtransferase) responsible for the conversion of $4 \mathrm{HB}$ to $4 \mathrm{HB}-\mathrm{CoA}$. The $H$. bluephagenesis mutant with the orfZ gene from Clostridium kluyveri with the T7-like expression system (MmP1) produced copolymer $\mathrm{P}(3 \mathrm{HB}-\mathrm{co}-4 \mathrm{HB})$ and the total content of the copolymer was $63 \%$ of dry weight (Chen et al. 2017). Shen et al. (2018) were also able to increase the accumulation rate of this copolymer up to $80 \%$ by using a more suitable promoter.

\section{Genetic modifications to avoid PHA degradation}

The intracellular activity of PHA depolymerase represents another challenge in PHA production. The enzyme is activated in the absence of a carbon source and degrades the accumulated PHA granules in the cell, resulting in the release of carbon and energy that the producer can use for its growth (Steinbüchel and Hein 2001). These enzymes are present on the surface of PHA granules (Rehm 2003) and, therefore the inactivation of intracellular PHA depolymerase by genetic engineering appears to be necessary to increase PHA recovery.

Povolo et al. (2015) deleted the phaZ1 gene encoding PHA depolymerase in the $C$. necator wild-type strain and their results suggest an increase in PHB accumulation during bacterial growth. However, knockout of the phaZ3 gene had no statistically significant effect on PHB accumulation, suggesting that the phaZ3 gene is unlikely to play a role in PHB degradation (Povolo et al. 2010). Kobayashi and Kondo (2019) also observed 2.9-fold higher PHB production by Rhodobacter sphaeroides HJ mutant with knockout of phaZ. Moreover, knockout of this gene associated with over-expression of PHB biosynthesis genes (phaA3, phaB2 and phaC1) caused 3.9-fold higher PHB production compared 
to the wild-type strain. Similar results have been reported by other authors focusing on the inactivation of mcl-PHA depolymerases (de Eugenio et al. 2007; Cai et al. 2008).

It should be noted that knockout of PHA depolymerase is not always associated with increased PHA recovery. Zhou et al. (2020) observed that the incorporation of the phaCl gene in $P$. putida KTc9n13 associated with disruption of the phaZ gene did not cause a significant increase in PHB accumulation. Also, Vo et al. (2015) did not observe a significant effect on increasing PHA production by damaging the phaZ gene in $P$. putida KT2440. Deletion of this gene caused 1.2-fold lower mcl-PHA production than wild-type strain. Salvachúa et al. (2020) did not observe a significant effect on PHA production by disruption of the phaZ gene in $P$. putida KT2440. Different results of these studies could be due to various cultivation conditions and genetic variability (difference of genotypes, deletion of the selected phaZ gene) (Zhou et al. 2020).

\section{Genome reduction for improving PHA production}

Genome reduction is a process in which appropriate deletion of selected genome fragments can increase producer growth, broaden the substrate specificity, improve energy metabolism, and increase the product yield and the genome stability (Fehér et al. 2007; Chi et al. 2019). The process eliminates a large proportion of the genome which is not important for the growth and production of PHA but consumes a lot of energy and carbon (Wang et al. 2018).

The $P$. mendocina NK-01 mutant with 14 large fragments removed and $7.7 \%$ genome reduction accumulated 11-fold higher mcl-PHA content than the wild-type strain (Fan et al. 2020). Deletion of 6 genes involved in the $\beta$-oxidation of fatty acids of $P$. medocina caused an increase of the dominant 3HD monomer to $90.01 \mathrm{~mol} \%$ and total mcl-PHA content to $58.6 \mathrm{~mol} \%$. Moreover, this modification increased the cell shape due to the higher accumulation of PHA granules in the intracellular space (Zhao et al. 2020). P. putida KT2440 with $4.12 \%$ reduction of the total genome size increased the amount of mcl-PHA up to 1.5-fold compared to the $P$. putida wild-type. Knockout of $g c d$ gene (glucose dehydrogenase gene) in the $P$. putida mutant caused a further $22.17 \%$ increase in PHA content (Liang et al. 2020). Wang et al. (2018) deleted genes involved in the production of flagella and pili in the $P$. putida KT2442 genome. The genome reduction by $1.2 \%$ resulted in accelerated mutant growth, no flagella production, reduced biofilm production and $45.6 \%$ higher PHA production than the original strain.

\section{Genetic modifications for simplify PHA isolation}

Down-stream processes affect the total cost of PHA production. The choice of isolation and purification steps is also related to the application of the biopolymer, because some steps may alter PHA properties or add undesirable biomass-derived compounds such as lipoproteins or toxins to the final product (Koller et al. 2013). Modification of the producer's genome by molecular biology tools may facilitate the process of isolating PHA granules from the cell (Borrero-de Acuña et al. 2017; Poblete-Castro et al. 2020) or reduce the number of isolation steps (Boynton et al. 1999; Rodríguez Gamero et al. 2018).

In recent years, the goal is to develop a programmable cell lysis system that allows the isolation of intracellular metabolites by autolysis of the production organism (Hajnal et al. 2016; Tamekou Lacmata et al. 2017; Borrero-de Acuña et al. 2017). Hori et al. (2002) used the phage holin-endolysin system in $B$. megaterium to release PHA from cells. Ultimately, approximately two-thirds of the accumulated PHA was released from the cells after induction of lysis by xylose. Cell lysis lasted approximately $30 \mathrm{~h}$. Martinez et al. (2011) used a similar system inducible by 3 -methyl benzoate in $P$. putida KT2440. It allowed the disruption of approximately $86 \%$ of the cells within $15 \mathrm{~h}$ and facilitated the subsequent extraction of PHA. Borrero-de Acuña et al. (2017) used toluic acid as the inductor in the phage holin-endolysin system. Although the use of the toluic acid inducer in $P$. putida KT2440 reduced the biomass by $25 \%$ during cultivation, mcl-PHA content was maintained at $30 \%$. After lysis, $95 \%$ of the bacterial cells were disrupted and approximately $75 \%$ of the total biopolymer was recovered during $16 \mathrm{~h}$. Hajnal et al. (2016) tested the implementation of the Lambda 
lysis system works in $H$. campaniensis. Spontaneous autolysis was observed after centrifugation of the cells and prolonged passive standing. The use of the lytic system has some disadvantages such as relatively long lysis time $(15-30 \mathrm{~h})$ and low amount of polymer released (75 - 86 \%) (Hori et al. 2002; Martinez et al. 2011; Borrero-de Acuña et al. 2017).

Another possibility is to modify the mechanisms responsible for maintaining cell wall integrity. OprF (porin F) is a protein responsible for the permeability and integrity of the cell wall (Chevalier et al. 2017), OprE (porin E) increases the diffusion of small ions (Jaouen et al. 2006), MscL (mechanosensitive channels of large conductance) acts as a safety valve opening pores in sudden hypo-osmotic shock, what is the last protection of the cell against their lysis due to osmotic shock (Colombo et al. 2003). Poblete-Castro et al. (2020) modified this system in $P$. putida by over-expressing the OprF and OprE, and deleting MscL. Due to hypertonic and hypotonic treatment, $95 \%$ of the cells were lysed within 3 h, with $94 \%$ mcl-PHA yield.

High-pressure homogenization (HPH) is one of the most widely used methods for cell lysis and is also one of the environmentally acceptable methods. However, its use releases large amounts of DNA, which increases the viscosity of the solution and complicates subsequent purification procedures (Ling et al. 1998; Tamer et al. 1998; Van Wegen et al. 1998). The decrease of viscosity is usually achieved by expensive processes, such as heat treatment or the addition of hypochlorite or commercially available nucleases (Koller et al. 2013). However, it is possible to insert the nuclease gene into a natural producer of PHA to reduce the viscosity and the cost of the downstream process. This method was also used by Boynton et al. (1999), which inserted the nuc gene encoding a nuclease from Staphylococcus aureus into natural PHA producers such as $C$. necator and $P$. putida. No negative effect on PHA growth and production was observed for both PHA producers. It was recorded the lower viscosity of the solution compared to wild-type strains treated with a commercial nuclease. The highest nuclease activity was observed for $P$. putida. Rodríguez Gamero et al. (2018) inserted the nuc gene into two native PHA producers, $C$. necator DSM 545 and Delftia acidovorans DSM 39. The gene was expressed in both producers, and the inserted gene did not affect the amount of produced biomass. In both cases, a higher amount of PHB was reported. The nuclease activity was higher in the $C$. necator mutant; the recombinant was used in the next experiments. The lower viscosity of the solution after HPH was observed compared to the original strain.

\section{Conclusion}

Genetic engineering of native PHA producers appears to be a suitable way to reduce the cost of PHA production. Molecular biology techniques can be used to broaden the range of substrates suitable for the production of PHAs, such as whey, molasses or crude glycerol. Morphology engineering as a new strategy for the construction of efficient microbial cell factories makes it possible to obtain larger and easier isolatable PHA granules from modified cells. Overexpression of selected genes involved in the PHA synthesis or knockout of the genes for specific metabolic pathways are associated with several advantages, such as increased PHA production, expansion of the range of produced biopolymers with different material properties adaptable to the intended purpose. Genetic modification also provides various ways to increase the number of monomers for PHA synthesis. The co-cultivation of two or more recombinant bacterial species appears to be promising due to cost reduction as well as the utilization of by-products. The overall resulting cost of PHAs can be reduced by a suitable isolation method through the implementation of selected self-destruction mechanisms based on the lytic system of bacteriophages or by porins. A key aspect of PHA production is use of by-products that presents an alternative to products primarily intended for human consumption. Production of PHAs can be introduced as a part of future biorefinery concept.

\section{Acknowledgements}

This work was supported by the Slovak Research and Development Agency under the contract No. APPV-18-0420. 


\section{Conflict of Interest}

The authors declare that there is no conflict of interest.

\section{References}

Akdoğan M, Çelik E (2020) Enhanced production of poly(3-hydroxybutyrate-co-3-hydroxyvalerate) biopolymer by recombinant Bacillus megaterium in fedbatch bioreactors. Bioprocess Biosyst. Eng. (in press).

Berezina N, Silvia MM (2014) Bio-based polymers and materials. In Luque $\mathrm{R}$, Lin C (Eds.), Renewable resources for biorefineries, vol. 27, The Royal Society of Chemistry, London, UK, pp. 1-28.

Borrero-De Acuña JM, Hidalgo-Dumont C, Pacheco N, Cabrera A, Poblete-Castro I (2017) A novel programmable lysozyme-based lysis system in Pseudomonas putida for biopolymer production. Sci. Rep. 7: e4373.

Bourab-Chibane L, Forquet V, Lantéri P, Clément Y, Léonard-Akkari L, Oulahal N, Degraeve P, Bordes C (2019) Antibacterial properties of polyphenols: characterization and qsar (quantitative structure-activity relationship) models. Front. Microbiol. 10: 829.

Boynton ZL, Koon JJ, Brennan EM, Clouart JD, Horowitz DM, Gerngross TU, Huisman GW (1999) Reduction of cell lysate viscosity during processing of poly(3hydroxyalkanoates) by chromosomal integration of the staphylococcal nuclease gene in Pseudomonas putida. Appl. Environ. Microbiol. 65: 1524-1529.

Cai L, Yuan MQ, Liu F, Jian J, Chen GQ (2009) Enhanced production of medium-chain-length polyhydroxyalkanoates (PHA) by PHA depolymerase knockout mutant of Pseudomonas putida KT2442. Bioresour. Technol. 100: 2265-2270.

Cardozo JRG, Bucheli RV, Sánchez CC, de la Mata Riesco I, Martínez ALM (2019) Engineering of Bacillus megaterium for improving PHA production from glycerol. Asia Pac. J. Mol. Biol. Biotechnol. 27: 64-72.

Cha G, Ha HS, Lee SK (2020) Metabolic engineering of Pseudomonas putida for the production of various types of short-chain-length polyhydroxyalkanoates from levulinic acid. Bioresour. Technol. 309: 123332.

Chen GQ, Jiang XR (2018) Engineering microorganisms for improving polyhydroxyalkanoate biosynthesis. Curr. Opin. Biotechnol. 53: 20-25.

Chen X, Yin J, Ye J, Zhang H, Che X, Ma Y, Li M, Wu LP, Chen GQ (2017) Engineering Halomonas bluephagenesis TD01 for non-sterile production of poly(3hydroxybutyrate-co-4-hydroxybutyrate). Bioresour. Technol. 244: 534-541.

Chevalier S, Bouffartigues E, Bodilis J, Maillot O, Lesouhaitier O, Feuilloley MGJ, Orange N, Dufour A, Cornelis P (2017) Structure, function and regulation of Pseudomonas aeruginosa porins. FEMS Microbiol. Rev. 41: 698-722.

Chi H, Wang X, Shao Y, Qin Y, Deng Z, Wang L, Chen S (2019) Engineering and modification of microbial chassis for systems and synthetic biology. Synth. Syst. Biotechnol. 4: 25-33.

Choi JI, Lee SY (1997) Process analysis and economic evaluation for poly(3-hydroxybutyrate) production by fermentation. Bioprocess Eng. 17: 335-342.

Choi SY, Rhie MN, Kim HT, Joo JC, Cho IJ, Son J, Jo SY, Sohn YJ, Baritugo K-A, Pyo J, Lee Y, Lee SY, Park SJ (2020) Metabolic engineering for the synthesis of polyesters: A 100-year journey from polyhydroxyalkanoates to non-natural microbial polyesters. Metab. Eng. 58: 47-81.

Colombo G, Marrink SJ, Mark AE (2003) Simulation of MscL gating in a bilayer under stress. Biophys. J. 84: 2331-2337.

De Eugenio LI, García P, Luengo JM, Sanz JM, Román JS, García JL, Prieto MA (2007) Biochemical evidence that phaZ gene encodes a specific intracellular medium chain length polyhydroxyalkanoate depolymerase in Pseudomonas putida KT2442: characterization of a paradigmatic enzyme. J. Biol. Chem. 282: 4951-4962.

Duarte de Oliveira GH, dos Santos Schneider AL, Vo MT, Ramsay JA, Ramsay BA (2020) Heterologous expression of Vitreoscilla hemoglobin in Pseudomonas putida KT2440 for the production of mcl-PHA in carbon-limited fermentations. Ind. Biotechnol. 16: 233-240.

Eggers J, Steinbüchel A (2013) Poly (3-hydroxybutyrate) degradation in Ralstonia eutropha H16 is mediated stereoselectively to (S)-3-hydroxybutyryl-CoA via crotonyl-CoA. J. Bacteriol. 195: 3213-3223.

Elhadi D, Lv L, Jiang XR, Wu H, Chen GQ (2016) CRISPRi engineering $E$. coli for morphology diversification. Metab. Eng. 38: 358-369.

Fan X, Zhang Y, Zheo F, Liu Y, Zhao Y, Wang S, Liu R, Yang C (2020) Genome reduction enhances production of polyhydroxyalkanoate and alginate oligosaccharide in Pseudomonas mendocina. Int. J. Biol. Macromol. 163: 2023-2031.

Favaro L, Basaglia M, Casella S (2019) Improving polyhydroxyalkanoate production from inexpensive carbon sources by genetic approaches: a review. Biofuel. Bioprod. Biorefin. 13: 208-227.

Fedeson DT, Saake P, Calero P, Nikel PI, Ducat DC (2020) Biotransformation of 2,4-dinitrotoluene in a phototrophic co-culture of engineered Synechococcus elongatus and Pseudomonas putida. Microb. Biotechnol. 13: 9971011.

Fehér T, Papp B, Pál C, Pósfai G (2007) Systematic genome reductions: theoretical and experimental approaches. Chem. Rev. 107: 3498-3513.

Flores-Sánchez A, Rathinasabapathy A, López-Cuellar MR, Vergara-Porras B, Pérez-Guevara F (2020) Biosynthesis of polyhydroxyalkanoates from vegetable oil under the co-expression of fadE and phaJ genes in Cupriavidus necator. Int. J. Biol. Macromol. 164: 1600-1607.

Goldford JE, Lu N, Bajić D, Estrela S, Tikhonov M, Sánchez-Gorostiaga A, Sergé D, Mehta P, Sanchez A (2018) Emergent simplicity in microbial community assembly. Science 361: 469-474.

Graf N, Altenbuchner J (2014) Genetic engineering of Pseudomonas putida KT2440 for rapid and high-yield 
production of vanillin from ferulic acid. Appl. Microbiol. Biotechnol. 98: 137-149.

Hajnal I, Chen X, Chen GQ (2016) A novel cell autolysis system for cost-competitive downstream processing. Appl. Microbiol. Biotechnol. 100: 9103-9110.

Hobmeier K, Löwe H, Liefeldt S, Kremling A, Pflüger-Grau K (2020) A Nitrate-blind P. putida strain boosts PHA production in a synthetic mixed culture. Front. Bioeng. Biotechnol. 8: 486.

Hori K, Kaneko M, Tanji Y, Xing XH, Unno H (2002) Construction of self-disruptive Bacillus megaterium in response to substrate exhaustion for polyhydroxybutyrate production. Appl. Microbiol. Biotechnol. 59: 211-216.

Jaouen T, Coquet L, Marvin-Guy L, Orange N, Chevalier S, De E (2006) Functional characterization of Pseudomonas fluorescens OprE and OprQ membrane proteins. Biochem. Biophys. Res. Commun. 346: 1048-1052.

Jiang XR, Chen GQ (2016) Morphology engineering of bacteria for bio-production. Biotechnol. Adv. 34: 435440.

Jiang XR, Yao ZH, Chen GQ (2017) Controlling cell volume for efficient PHB production by Halomonas. Metab. Eng. 44: 30-37.

Jung HR, Jeon JM, Yi DH, Song HS, Yang SY, Choi TR, Bhatia SK, Yoon JJ, Kim YG, Brigham CJ, Yang YH (2019) Poly(3-hydroxybutyrate-co-3-hydroxyvalerate-co3- hydroxyhexanoate) terpolymer production from volatile fatty acids using engineered Ralstonia eutropha. Int. J. Biol. Macromol. 138: 370-378.

Keshavarz T, Roy I (2010) Polyhydroxyalkanoates: bioplastics with a green agenda. Curr. Opin. Microbiol. 13: 321-326.

Kobayashi J, Kondo A (2019) Disruption of poly (3hydroxyalkanoate) depolymerase gene and overexpression of three poly(3-hydroxybutyrate) biosynthetic genes improve poly (3-hydroxybutyrate) production from nitrogen rich medium by Rhodobacter sphaeroides. Microb. Cell Fact. 18: 40.

Koller M, Bona R, Braunegg G, Hermann C, Horvat P, Kroutil M, Martinz J, Neto J, Pereira L, Varila P (2005) Production of polyhydroxyalkanoates from agricultural waste and surplus materials. Biomacromolecules 6: 561565.

Koller M, Hesse P, Bona R, Kutschera C, Atlić A, Braunegg $G$ (2007) Potential of various archae- and eubacterial strains as industrial polyhydroxyalkanoate producers from whey. Macrobiol. Biosci. 7: 218-226.

Koller M, Niebelschütz H, Braunegg G (2013) Strategies for recovery and purification of poly[(R)-3-hydroxyalkanoates] (PHA) biopolyesters from surrounding biomass. Eng. Life Sci. 13: 549-562.

Laycock B, Halley P, Pratt S, Werker A, Lant P (2013) The chemomechanical properties of microbial polyhydroxyalkanoates. Prog. Polym. Sci. 38: 536-583.

Leong YK, Show PL, Ooi CW, Ling TC, Lan JCW (2014) Current trends in polyhydroxyalkanoates (PHAs) biosynthesis: Insights from the recombinant Escherichia coli. J. Biotechnol. 180: 52-65.
Liang P, Zhang Y, Xu B, Zhao Y, Liu X, Gao W, Yang C, Wang S, Liu R (2020) Deletion of genomic islands in the Pseudomonas putida KT2440 genome can create an optimal chassis for synthetic biology applications. Microb. Cell Fact. 19: 70.

Ling Y, Wong HH, Thomas CJ, Williams DRG, Middelberg APJ (1998) Pilot-scale extraction of PHB from recombinant $E$. coli by homogenization and centrifugation. Bioseparation 7: 9-15.

Ling C, Qiao GQ, Shuai BW, Olavarria K, Yin J, Xiang R, Song KN, Shen YH, Guo Y, Chen GQ (2018) Engineering $\mathrm{NADH} / \mathrm{NAD}^{+}$ratio in Halomonas bluephagenesis for enhanced production of polyhydroxyalkanoates (PHA). Metab. Eng. 49: 275-286.

Liu F, Jian J, Shen X, Chung A, Chen J, Chen GQ (2011) Metabolic engineering of Aeromonas hydrophila 4AK4 for production of copolymers of 3-hydroxybutyrate and medium-chain-length 3-hydroxyalkanoate. Bioresour. Technol. 102: 8123-8129.

Liu K, Liu G, Zhang Y, Ding J, Weng W (2014) Engineering of a $D$-xylose metabolic pathway in Eutropha W50. Acta Microbiol. Sin. 54: 42-52.

Liu Y, Yang S, Jia X (2020) Construction of a "nutrition supply-detoxification" coculture consortium for medium-chain-length polyhydroxyalkanoate production with a glucose-xylose mixture. J. Ind. Microbiol. Biotechnol. 47: 343-354.

Lu J, Tappel R, Nomura CT (2009) Mini-review: biosynthesis of poly (hydroxyalkanoates). Polym. Rev. 49: 226-248.

Lu X, Liu G, Wang Y, Ding J, Weng W (2013) Engineering of an $L$-arabinose metabolic pathway in Ralstonia eutropha W50. Acta Microbiol. Sin. 53: 1267-1275.

Magdouli S, Brar SK, Blais JF, Tyagi RD (2015) How to direct the fatty acid biosynthesis towards polyhydroxyalkanoates production? Biomass Bioenergy 4: 268-279.

Martinez V, Garcia P, Garcia JL, Prieto MA (2011) Controlled autolysis facilitates the polyhydroxyalkanoate recovery in Pseudomonas putida KT2440. Microb. Biotechnol. 4: 533-547.

Matsusaki H, Abe H, Taguchi K, Fukui T, Doi Y (2000a) Biosynthesis of poly(3-hydroxybutyrate-co-3hydroxyalkanoates) by recombinant bacteria expressing the PHA synthase gene phaCl from Pseudomonas sp. 613. Appl. Microbiol. Biotechnol. 53: 401-409.

Matsusaki H, Abe H, Doi Y (2000b) Biosynthesis and properties of poly(3-hydroxsybutyrate-co-3hydroxyalkanoates) by recombinant strains of Pseudomonas sp. 61-3. Biomacromolecules 1: 17-22.

Matsumoto K, Matsusaki H, Taguchi S, Seki M, Doi Y (2001) Cloning and characterization of the Pseudomonas sp. 61-3 phaG gene involved in polyhydroxyalkanoate biosynthesis. Biomacromolecules 2: 142-147.

Noda I, Green PR, Satkowski MM, Schechtman LA (2005) Preparation and properties of a novel class of polyhydroxyalkanoate copolymers. Biomacromolecules 6: 580-586.

Ouyang P, Wang H, Hajnal I, Wu Q, Guo Y, Chen GQ (2018) Increasing oxygen availability for improving poly(3- 
hydroxybutyrate) production by Halomonas. Metab. Eng. 45: 20-31.

Pantazaki AA, Papaneophytou CP, Pritsa AG, LiakopoulouKyriakides M, Kyriakidis DA (2009) Production of polyhydroxyalkanoates from whey by Thermus thermophilus HB8. Process Biochem. 44: 847-853.

Park SJ, Jang YA, Noh W, Oh YH, Lee H, David Y, Baylon, MG, Shin J, Yang JE, Choi SY, Lee SH, Lee SY (2015) Metabolic engineering of Ralstonia eutropha for the production of polyhydroxyalkanoates from sucrose. Biotechnol. Bioeng. 112: 638-643

Philip S, Keshavarz T, Roy I (2007) Polyhydroxyalkanoates: biodegradable polymers with a range of applications. J. Chem. Technol. Biotechnol. 82: 233-247.

Poblete-Castro I, Aravena-Carrasco C, Orellana-Saez M, Pacheco N, Cabrera A, Borrero-de Acuña JM (2020) Engineering the osmotic state of Pseudomonas putida KT2440 for efficient cell disruption and downstream processing of poly(3-hydroxyalkanoates). Front. Bioeng. Biotechnol. 8: 161.

Pötter M, Steinbüchel A (2005) Poly(3-hydroxybutyrate) granule-associated proteins: impacts on poly(3hydroxybutyrate) synthesis and degradation. Biomacromolecules 6: 552-560.

Povolo S, Toffano P, Basaglia M, Casella S (2010) Polyhydroxyalkanoates production by engineered Cupriavidus necator from waste material containing lactose. Bioresour. Technol. 101: 7902-7907.

Povolo S, Basaglia M, Fontana F, Morelli A, Sergio C (2015) Poly(hydroxyalkanoate) production by Cupriavidus necator from fatty waste can be enhanced by phaZ1 inactivation. Chem. Biochem. Eng. Q. 29: 67-74.

Rehm BHA (2003) Polyester synthases: natural catalysts for plastics. Biochem. J. 376: 15-33.

Rodríguez Gamero JE, Favaro L, Pizzocchero V, Lomolino G, Basaglia M, Casella S (2018) Nuclease expression in efficient polyhydroxyalkanoates-producing bacteria could yield cost reduction during downstream processing. Bioresour. Technol. 261: 176-181.

Salvachúa D, Rydzak T, Auwae R, De Capite A, Black BA, Bouvier JT, Cleveland NS, Elmore JR, Huenemann JD, Katahira R, Michener WE, Peterson DJ, Rohrer H, Vardon DR, Beckham GT, Guss AM (2020) Metabolic engineering of Pseudomonas putida for increased polyhydroxyalkanoate production from lignin. Microbiol. Biotechnol. 13: 290-298.

Sauer U, Canonaco F, Heri S, Perrenoud A, Fischer E (2004) The soluble and membrane-bound transhydrogenases UdhA and PntAB have divergent functions in NADPH metabolism of Escherichia coli. J. Biol. Chem. 279: 6613-6619.

Shen R, Yin J, Ye JW, Xiang RJ, Ning ZY, Huang WZ, Chen GQ (2018) Promoter engineering for enhanced P(3HB-co4HB) production by Halomonas bluephagenesis. ACS Synth. Biol. 7: 1897-1906.

Shen R, Ning YZ, Lan YX, Chen JC, Chen GQ (2019) Manipulation of polyhydroxyalkanoate granular sizes in Halomonas bluephagenesis. Metab. Eng. 54: 117-126.

Shi LL, Da YY, Zheng WT, Chen GQ, Li ZJ (2020) Production of polyhydroxyalkanoate from acetate by metabolically engineered Aeromonas hydrophilia. J. Biosci. Bioeng. 130: 290-294.

Solaiman DKY, Ashby RD, Foglia TA, Marmer WN (2006) Conversion of agricultural feedstock and coproducts into poly(hydroxyalkanoates). Appl. Microbiol. Biotechnol. 71: 783-789.

Steinbüchel A (1991) Polyhydroxyalkanoic acid. In Byrom D (Ed.), Biomaterials: Novel Materials from Biological Sources, Palgrave Macmillan, UK, London, pp. 123-213.

Steinbüchel A, Hein S (2007) Biochemical and molecular basis of microbial synthesis of polyhydroxyalkanoates in microorganisms. Adv. Biochem. Eng. Biotechnol. 71: 81-123.

Tamekou Lacmata S, Yao L, Xian M, Liu H, Kuiate JR, Liu H, Feng X, Zhao G (2017) A novel autolysis system controlled by magnesium and its application to poly (3hydroxypropionate) production in engineered Escherichia coli. Bioengineered 8: 594-599.

Tamer IM, Moo-Young M, Christi Y (1998) Disruption of Alcaligenes latus for recovery of $\operatorname{poly}(\beta$ hydroxybutyric acid): Comparison of high-pressure homogenization, bead milling, and chemically induced lysis. Ind. Eng. Chem. Res. 37: 1807-1814.

Tan D, Wu Q, Chen JC, Chen GQ (2014a) Engineering Halomonas TD01 for the low-cost production of polyhydroxyalkanoates. Metab. Eng. 26: 34-47.

Tan GYA, Chen CL, Li L, Ge L, Wang L, Razaad IMN, Li Y, Zheo L, Mo Y, Wang JY (2014b) Start a research on biopolymer polyhydroxyalkanoate $(\mathrm{PHa})$ : A review. Polymers 6: 706-754.

Tan IKP, Foong CP, Tan HT, Lim H, Zain NAA, Tan ZC, Hoh CC, Sudesh K (2020) Polyhydroxyalkanoate (PHA) synthase genes and PHA-associated gene clusters in Pseudomonas spp. and Janthinobacterium spp. isolated from Antarctica. J. Biotechnol. 313: 18-28.

Tang R, Weng C, Peng X, Han Y (2020) Metabolic engineering of Cupriavidus necator $\mathrm{H} 16$ for improved chemoautotrophic growth and PHB production under oxygen-limiting conditions. Metab. Eng. 61: 11-23.

Tian J, Sinskey AJ, Stubbe J (2005) Detection of intermediates from the polymerization reaction catalyzed by a D302A mutant of class III polyhydroxyalkanoates (PHA) synthase. Biochemistry 44: 1495-1503.

Troschl C, Meixner K, Drosg B (2017) Cyanobacterial PHA production-review of recent advances and a summary of three years' working experience running a pilot plant. Bioengineering (Basel) 4: 26.

Van Wegen RJ, Ling Y, Middelberg APJ (1998) Industrial production of polyhydroxyalkanoates using Escherichia coli: An economic analysis. Chem. Eng. Res. Des. 76: 417-426.

Vo MT, Ko K, Ramsay B (2015) Carbon-limited fed-batch production of medium-chain-length polyhydroxyalkanoates by a phaZ-knockout strain of Pseudomonas putida KT2440. J. Ind. Microbiol. Biotechnol. 42: 637-646.

Wang J, Ma W, Wang Y, Lin L, Wang T, Wang Y, Li Y, Wang X (2018) Deletion of 76 genes relevant to flagella and pili formation to facilitate polyhydroxyalkanoate 
production in Pseudomonas putida. Appl. Microbiol. Biotechnol. 102: 10523-10539.

Webster DA (1988) Structure and function of bacterial hemoglobin and related proteins. Adv. Inorg. Biochem. 7: 245-265.

Wu H, Fan Z, Jiang X, Chen J, Chen G-Q (2016) Enhanced production of polyhydroxybutyrate by multiple dividing E. coli. Microb. Cell Fact. 15: 128.

Yang S, Li S, Jia X (2019) Production of medium chain length polyhydroxyalkanoate from acetate by engineered Pseudomonas putida KT2440. J. Ind. Microbiol. Biotechnol. 46: 793-800.

Yellore V, Desai A (1998) Production of poly-3hydroxybutyrate from lactose and whey by Methylobacterium sp. ZP24. Lett. Appl. Microbiol. 26: 391-394.

Yu LP, Yan X, Zhang X, Chen XB, Wu Q, Jiang XR, Chen GQ (2020) Biosynthesis of functional polyhydroxyalkanoates by engineered Halomonas bluephagenesis. Metab. Eng. 59: 119-130.
Zhang B, Carlson R, Srienc F (2006) Engineering the monomer composition of polyhydroxyalkanoates synthetized in Saccharomyces cerevisiae. Appl. Environ. Microbiol. 72: 536-543.

Zhao F, Gong T, Liu X, Fan X, Huang R, Ma T, Eang S, Gao W, Yang C (2019) Morphology engineering for enhanced production of medium-chain-length polyhydroxyalkanoates in Pseudomonas mendocina NK-01. Appl. Microbiol. Biotechnol. 103: 1713-1724.

Zhao F, He F, Liu X, Shi J, Liang J, Wang S, Yang C, Liu R (2020) Metabolic engineering of Pseudomonas mendocina NK-01 for enhanced production of medium-chain-length polyhydroxyalkanoates with enriched content of the dominant monomer. Int. J. Biol. Macromol. 154: 1596-1605.

Zhou, Y, Lin L, Wang H, Zhang Z, Zhou J, Jiao N (2020) Development of a CRISPR/Cas9n-based tool for metabolic engineering of Pseudomonas putida for ferulic acid-to polyhydroxyalkanoate bioconversion. Commun. Biol. 3: 98. 